\title{
Factors affecting synthesis of single wall carbon nanotubes in arc discharge
}

\author{
Michael Keidar \\ Department of Aerospace Engineering, University of Michigan, Ann Arbor, MI 48109, USA \\ E-mail: keidar@umich.edu
}

Received 17 August 2006, in final form 6 October 2006

Published 4 April 2007

Online at stacks.iop.org/JPhysD/40/2388

\begin{abstract}
In this paper a model of single wall carbon nanotube (SWNT) interactions with thermal plasma is developed. Several effects such as momentum, charge and energy transfer between the SWNT and plasma are considered. It is shown that the SWNT charge and potential with respect to the plasma, as well as SWNT aspect ratio, depend on plasma density and electric field in the interelectrode gap. For instance, the SWNT charge changes from negative to positive value with electron density decrease. This model suggests several ways to improve the controllability of SWNT synthesis in arc discharge, such as an increase in plasma density and the application of an electric field. It is predicted that higher plasma density in the SWNT formation region and electric field lead to the formation of SWNT with a very high aspect ratio.
\end{abstract}

\section{Introduction}

Research focused on carbon nanotubes (CNTs) has led to significant progress in synthesis techniques and a variety of CNT applications. Tremendous interest in carbon nanotubes has been stimulated by their unique mechanical, thermal and electrical properties. Nowadays applications of CNTs include field-emission displays [1,2], nanoelectronics [3,4] hydrogen storage [5], chemical gas sensors [6] etc. Plasmaenhanced methods of CNT synthesis are one of the most efficient and precise tools of fabrication of the carbon-based nanostructures $[7,8]$. However the processes of nucleation and growth of nanotubes are not completely understood. Thus, the full potential of CNTs for the aforementioned and other future applications cannot be realized until the synthesis techniques are optimized and ways to control CNTs characteristics are determined.

Several techniques have been developed for CNT synthesis such as arc discharge, chemical vapour deposition (CVD) and laser ablation [9-12]. Arc discharge is the most practical method of single wall carbon nanotube (SWNT) synthesis. CNTs produced by the arc discharge technique have fewer structural defects than those produced by low temperature techniques. They are perfectly straight as compared with the kinked type obtained by CVD processes. Most likely, this is due to fast growth that prevents defect formation. In addition, it was shown that among the several methods of SWNT production, nanotubes produced by arc discharge have the lowest time degradation of emission capability [13] which is very important for field-emission applications.

It is of general consensus that key parameter for nanotube growth in the arc discharge is the anode erosion rate. Part of the ablated material is deposited on the cathode. Two different textures and morphologies can be observed in the cathode deposit, such as a grey outer shell and a dark-soft inner core deposit. Multi wall nanotubes (MWNT) as well as graphitic particles are typically found in the inner core [14]. SWNTs produced by the anodic arc discharge are found in a 'collaret' around the cathode deposit, clothlike soot suspended in the chamber walls and the weblike structure suspended between the cathode and the walls [16]. Dependent on gas background and arc conditions MWNTs [15] and SWNTs are produced in arc discharge $[16,17]$. Among the other factors, current conductivity across deposit may affect SWNT formation. In fact, recently, the effect of current conductivity across the SWNT sample on ignition was investigated [18]. This effect may play a role during SWNT synthesis.

Recent progress in the arc discharge method of SWNT synthesis was motivated by Journet et al [19], who showed that SWNTs can be produced in large quantities. The issues related to large scale and high purity synthesis of SWNT by arc discharge are the most important objectives nowadays [20-24]. It is known that SWNT growth in arc discharge depends on the background pressure in helium and argon atmosphere. In the $\mathrm{He}-\mathrm{Ar}$ mixture it was found that the argon mole fraction 
affects the SWNT diameter [25], while the SWNT diameter was found to be fairly independent of pressure in the pure helium environment [16]. However, the overall relative density of the carbon species, and not necessarily a specific buffer gas, are critical for nanotube growth [26]. In addition to the SWNT diameter, two other parameters are important for SWNT applications, namely chirality and aspect ratio [27]. The chiral angle determines whether SWNT has metallic or semiconductor electrical conductivity [28].

Several models of a cathodic carbon arc were developed in the past dealing with electrode phenomena [29-31] or interelectrode plasma [32]. Recently a one-dimensional model (in the axial direction) of SWNT formation was developed and the SWNT growth rate in an anodic arc discharge was calculated [26]. According to existing model predictions nanotube formation occurs in the region of relatively small plasma temperature (1300-1800 K [33]) where carbon reacts to form large molecules and clusters. In some models [26] the anode temperature was assumed, while recent work showed that the anode temperature depends on the gas pressure [34]. No detailed model for the relationship between the discharge parameters and SWNT formation was developed as mentioned in a recent review paper [35]. A model of SWNT interaction with discharge plasma and SWNT formation in the cathode region (collaret) was developed [36]. It was shown that under certain conditions SWNT can be deposited on the cathode surface. This process depends on SWNT charging in the arc plasma. In turn, the charging phenomena depend on the electron temperature.

The ability to control SWNT properties is important for many practical applications. While, in general, arc discharge technique is considered to offer poor flexibility, it is primarily a result of limited understanding of the SWNT synthesis mechanism. Among SWNT properties of interest are SWNT radius, the chirality and length. It was mentioned above that the SWNT radius can be controlled by the type of the gas in the chamber while the gas pressure leads to fairly constant radius. The chiral angle determines whether the CNT has metallic or semiconductor electrical conductivity. In particular, there is tremendous interest in synthesis of long SWNT, which will enable new types of MEMS/NEMS systems, such as microelectric motors and can act as a nanoconducting cable [37]. Recently growth of $4 \mathrm{~cm}$ long SWNT was reported [37]. The question is, what are the theoretical and the practical limits for the SWNT length and what are the factors affecting the length of SWNT growing by the arc discharge technique. In order to answer these questions one should construct a detailed model of SWNT synthesis. A development of the full picture goes beyond the framework of this paper and we will concentrate on several aspects of SWNT synthesis and peculiarities of the arc discharge technique as well as possible SWNT-plasma interactions.

In recent years several models of CNT formation in an arc discharge have been proposed. Gamaly and Ebbesen [38] proposed that the bimodal carbon velocity distribution (ions with drift velocity and isotropic neutrals) determines the nanotube creation process near the cathode. They suggested that isotropic distribution leads to fullerene formation while directed flux results in nanotubes. Iijima et al [39] proposed an open-ended growth model. In this model carbon atoms and small carbon clusters add on to the reactive dangling bonds at the edges of the open-ended nanotubes. Other researchers argue that carbon nanotubes are elongated by electrostatic forces along the electric field near the cathode [40,41]. However it seems like a high-resolution transmission electron microscopy analysis does not support this hypothesis. Alternatively a two-step growth model has been proposed [42]. According to this model, different carbon structures are formed first. Then, during the cooling process, the graphitization occurs from the surface towards the interior of the assemblies. Several workers developed a growth model of SWNT explaining the root-growth of nanotube bundles emerging from catalyst particles [35]. These models include a catalyst phase diagram of carbon-metal. Most essential aspects of this model were described by Moravsky et al [14].

Apparently, chemical processes involved in SWNT growth determine the length of the nanotubes. In this paper we consider an additional factor that may affect SWNT synthesis, namely SWNT seed residence time in the region of preferable growth. It will be shown that this factor has a very strong effect on the SWNT growth rate and thus on the SWNT properties.

\section{The model of SWNT interactions with interelectrode plasma}

In this section, we briefly describe a model of SWNT interactions with interelectrode plasma and consider SWNT synthesis in the interelectrode region. Typically in the interelectrode gap of the arc discharge plasma temperature varies from about $5000 \mathrm{~K}$ in the centre of the channel down to $1000 \mathrm{~K}$ at the periphery $[16,35]$. The probability of atomic collisions and therefore nanotube seed formation is higher in the centre of the interelectrode gap, i.e. in the region with the highest carbon atoms and ions density. Recall that the SWNT seeds formed in the plasma are subject to interaction with plasma particles that include charge, momentum and energy transfer. As a result of these interactions high heat fluxes may lead to overheating and prevention of the formation of the stable nanostructures. Thus, nanotube formation occurs in the region of relatively low plasma temperature (1300$1800 \mathrm{~K}$ [34]) where carbon reacts to form large molecules and clusters. The length of that region is about a few $\mathrm{mm}$ [35].

Carbon clusters diffuse with diffusion coefficient $D_{\text {SWNT }}$ from the region of origin without any chemical reactions [43]. In the diffusion approximation one can determine the diffusion coefficient of carbon clusters and SWNT as follows [43]:

$D_{\mathrm{SWNT}}=\frac{4}{3} \frac{\sqrt{2}}{\pi^{3 / 2}}\left(\frac{1}{m_{\mathrm{SWNT}}}+\frac{1}{m_{\mathrm{He}}}\right)^{1 / 2} \frac{(k T)^{3 / 2}}{p\left(d_{\mathrm{SWNT}}+d_{\mathrm{He}}\right)^{2}}$,

where $m_{\mathrm{SWNT}}, m_{\mathrm{He}}$ are SWNT and He molecular masses, $T$ is the plasma temperature (it is assumed that the plasma is in a local thermodynamic equilibrium (LTE), $[16,34]$ ), $p$ is the pressure and $d_{\mathrm{SWNT}}$ and $d_{\mathrm{He}}$ are the effective diameters of the SWNT seed and He molecular respectively. In this formulation the radial diffusion velocity of SWNT seed can be estimated as: $V_{\mathrm{SWNT}} \sim D_{\mathrm{SWNT}} / R_{\mathrm{a}}$, where $R_{\mathrm{a}}$ is the anode radius, which is the characteristic dimension of the plasma region. In addition, an initial SWNT velocity can be estimated from experimental 
Table 1. Typical plasma and SWNT parameters.

\begin{tabular}{|c|c|}
\hline Measured SWNT velocity & $0.01-0.5 \mathrm{~m} \mathrm{~s}^{-1}[43]$ \\
\hline He pressure & 100-500 Torr \\
\hline $\begin{array}{l}\text { Spatial scale of the region of SWNT } \\
\text { formation }\end{array}$ & $\sim 1 \mathrm{~mm}[35]$ \\
\hline $\begin{array}{l}\text { SWNT temperature in the region of } \\
\text { formation }\end{array}$ & $1500 \mathrm{~K}$ \\
\hline Plasma temperature & $0.5 \mathrm{eV}[16,34]$ \\
\hline
\end{tabular}

measurements [43]. The typical range of SWNT velocities is presented in table 1 .

The charge transfer from the plasma to SWNT is due to electron and ion fluxes to the SWNT seed and due to thermoionic emission from the hot SWNT:

$$
\frac{\mathrm{d} Q_{\mathrm{SWNT}}}{\mathrm{d} t}=I_{\mathrm{i}}-I_{\mathrm{e}}+I_{\mathrm{em}},
$$

where $Q_{\mathrm{SWNT}}$ is SWNT charge, $I_{\mathrm{i}}, I_{\mathrm{e}}$ and $I_{\mathrm{em}}$ are ion, electron and thermoionic current, respectively. The electron current is given by: $I_{\mathrm{e}}=S j_{\mathrm{e}}$, where $S$ is SWNT surface area. The electron current density absorbed by SWNT depends upon SWNT potential with respect to the surrounding plasma: $j_{\mathrm{e}}=$ $j_{\mathrm{eo}} \exp \left(-e \varphi_{\mathrm{SWNT}} / k T\right)$ if $\varphi_{\mathrm{SWNT}}<0$ and $j_{\mathrm{e}}=j_{\mathrm{eo}} \exp (1+$ $\left.e \varphi_{\mathrm{SWNT}} / k T\right)$ in the opposite case, where $j_{\mathrm{eo}}$ is the electron thermal current density, $\varphi_{\mathrm{SWNT}}$ is the SWNT potential with respect to the plasma. The ion current density at the SWNT surface is given by [36]: $j_{\mathrm{i}}=j_{\text {io }}(1+\alpha)$ if $\alpha \geqslant 0$ and $j_{\mathrm{i}}=j_{\mathrm{io}}$ if $-1<\alpha<0$ and $j_{\mathrm{i}}=0$ if $\alpha<-1$, where $\alpha=-2 e \varphi_{\mathrm{SWNT}} / m_{\mathrm{i}} V_{\mathrm{i}}^{2}$ and $j_{\mathrm{io}}=e n_{\mathrm{e}} V_{\mathrm{i}}$ is the ion current density in the plasma and $V_{\mathrm{i}}$ is the ion velocity.

The current of thermoionic emission is given by the Richardson-Duschman equation:

$$
I_{\mathrm{em}}=S A T_{\mathrm{s}}^{2} \exp \left(-\frac{e\left[\Phi+\varphi_{\mathrm{SWNT}}\right]}{k T_{\mathrm{s}}}\right),
$$

where $\Phi$ is the work function, $T_{\mathrm{S}}$ is the SWNT temperature (see table 1).

Following [36, 44], the electric capacitance of the cylindrical particle is calculated as $C=4 \pi \varepsilon_{\mathrm{o}}(L / \ln (2 L / a))$, where $L$ is the SWNT length and $a$ is the SWNT radius $(a=1.4 \mathrm{~nm},[16])$. The capacitance does not depend on the inner radius of SWNT since it is calculated between the SWNT and the surrounding plasma. In addition, we take into account that SWNT is a conductor (having either metallic or semiconductor properties), thus SWNT charge is equal $Q_{\mathrm{SWNT}}=C \varphi_{\mathrm{SWNT}}$.

Densities of the carbon species are determined by the anode ablation rate. The maximum density of carbon atoms/molecules in the interelectrode gap can be estimated from the vapour pressure. For instance, in the case of $\mathrm{He}$ pressure of about 500 Torr the resulting anode temperature is about $3270 \mathrm{~K}$ and the anode erosion rate is about $3 \mathrm{~mm}^{3} \mathrm{~s}^{-1}[16,34]$. Based on these calculations one can estimate the upper limit of the carbon species density as: $n_{\mathrm{C}}=P_{\mathrm{v}} /(k T)$, where $P_{\mathrm{v}}$ is the vapour pressure at the anode. The density decreases due to plasma expansion in the radial direction and, typically, plasma density has its highest value along the arc discharge centreline [45].

SWNT growth rate is determined by carbon atoms and ions precipitation to the nanotube surface and chemical reactions,

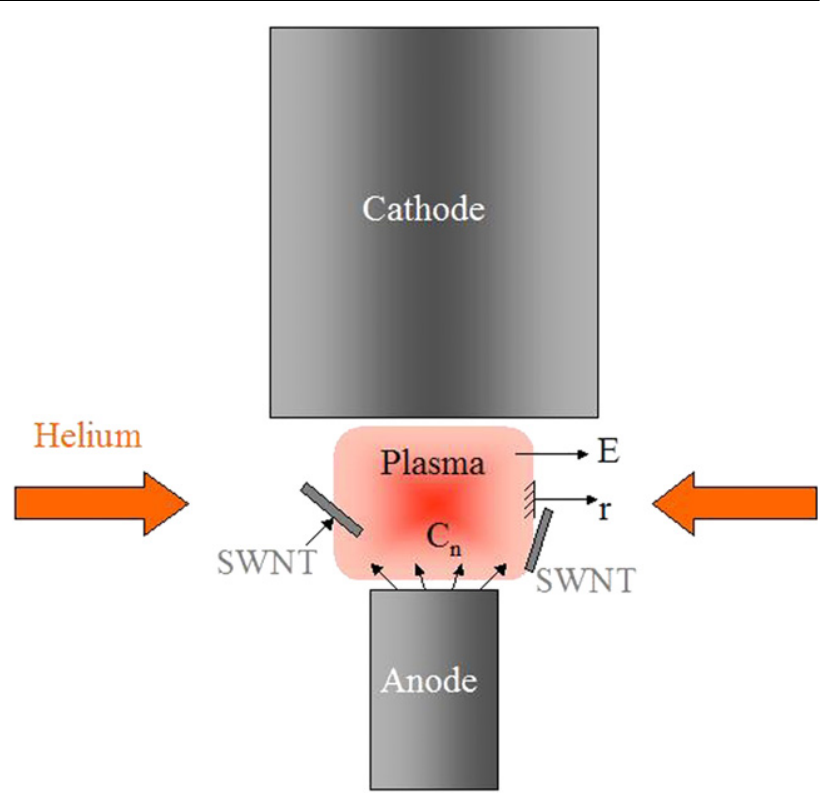

Figure 1. Schematic of the arc discharge and simulation geometry. (This figure is in colour only in the electronic version)

which depends on the density of carbon species in the vicinity of SWNT and the electron temperature. It is assumed that the influx of carbon ions and atoms to SWNT causes an increase in SWNT length. We further assume that growing SWNT has $\mathrm{C}-\mathrm{C}$ spacing of about $1.4^{\circ} \mathrm{A}$ [34]. Flux of the carbon atoms to the SWNT surface can be calculated as follows:

$$
\Gamma=0.25 n_{\mathrm{C}} \sqrt{\frac{8 k T}{\pi m}} .
$$

The SWNT interaction with plasma in the interelectrode region leads to momentum transfer, which can be accounted as follows:

$$
M_{\mathrm{SWNT}} \frac{\mathrm{d} V_{\mathrm{SWNT}}}{\mathrm{d} t}=F_{\mathrm{D}}+Q_{\mathrm{SWNT}} E,
$$

where $F_{\mathrm{D}}$ is the drag force, $F_{\mathrm{D}}=\frac{1}{4} \pi \rho d_{\mathrm{SW}}^{2}\left(V_{\mathrm{i}}-V_{\mathrm{SWNT}}\right)^{2}$ [36], $\rho$ is the plasma density, and $E$ is the electric field in the region of the SWNT formation. Equation (5) is supplemented by the equation for SWNT trajectory: $\mathrm{d} r / \mathrm{d} t=V_{\mathrm{SWNT}}$. The initial velocity of $S W N T, V_{\mathrm{SWNT}}(0)$, is calculated from equation (1). Electric field in this region can be created, for instance, by placing the biasing electrode. In this paper, we will explore this effect by calculating the SWNT-plasma interaction under different plasma conditions. In the next section, it will be shown that momentum transfer can be controlled by an electric field dependent on the SWNT charge.

It is assumed that the plasma parameters such as plasma density, temperature, carbon neutral density and the SWNT temperature are known from previously developed models. Based on these typical values, the SWNT velocity, charge distribution and the SWNT aspect ratio were calculated. These results are presented in the next section.

\section{Results and discussions}

The calculated SWNT velocity distribution in the radial direction (for coordinate system see figure 1) is shown in 


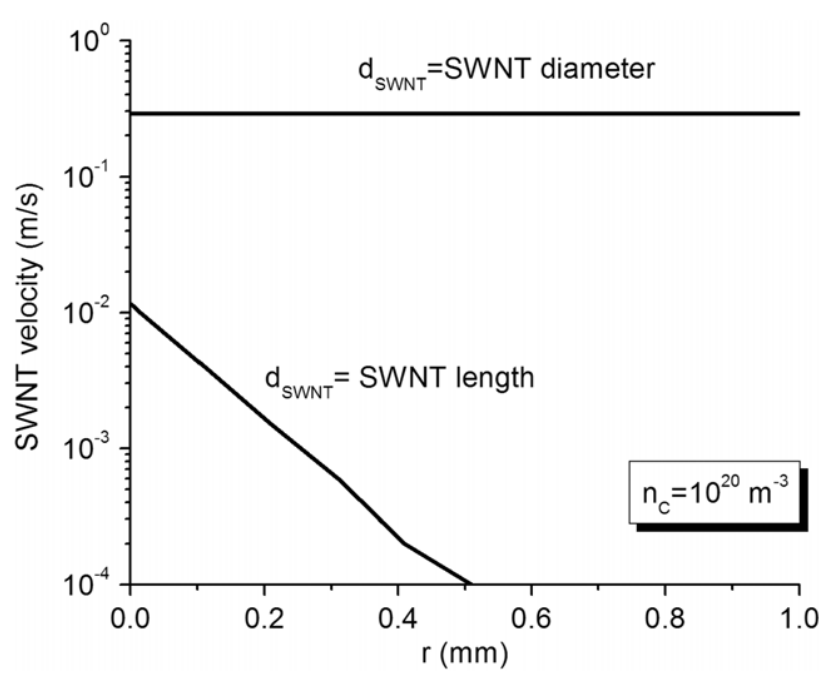

Figure 2. SWNT velocity distribution with characteristic SWNT size as a parameter.

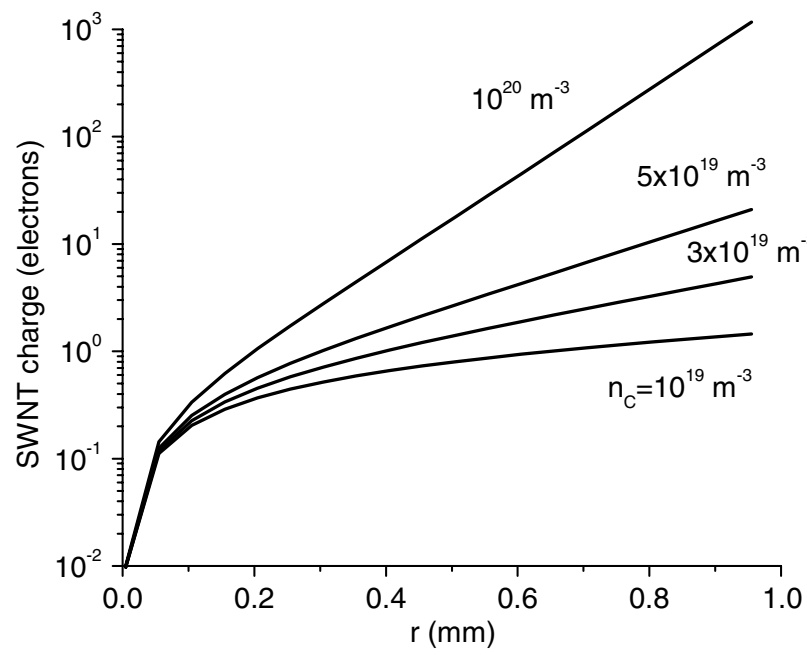

Figure 3. SWNT charge as a function of distance from the SWNT seed formation position. Electron density is $10^{10} \mathrm{~m}^{-3}$.

figure 2. Diffusion across the interelectrode plasma region leads to dependence of the SWNT velocity on the characteristic SWNT size due to momentum transfer. In figure 2 the characteristic SWNT size is used as a parameter. Two limited cases with the SWNT length and diameter as characteristic the SWNT size are shown. One can see that the SWNT diffusion velocity is about $0.3 \mathrm{~m} \mathrm{~s}^{-1}$ in the case of the characteristic size equal to the SWNT diameter, while this velocity is much smaller in the case of the characteristic size equal to SWNT length. During the diffusion, the SWNT length varies due to the SWNT growth as will be shown below. It should be pointed out that the measured SWNT diffusion velocity is about $0.01-0.5 \mathrm{~m} \mathrm{~s}^{-1}$ (see table 1), which is consistent with these predictions.

The SWNT charge distribution in the radial direction is shown in figure 3 with carbon species density as a parameter. In these calculations it was assumed that SWNT growth is terminated when SWNT leaves the formation region, which has a thickness of about $1 \mathrm{~mm}$. The SWNT relative length (aspect ratio) is shown in figure 4 with carbon species density as

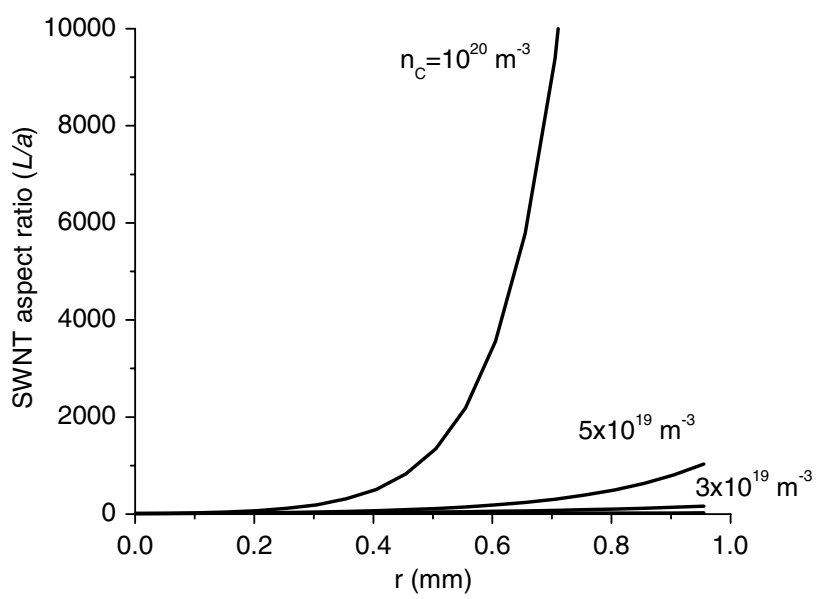

Figure 4. SWNT aspect ratio as a function of distance from the SWNT seed formation position.

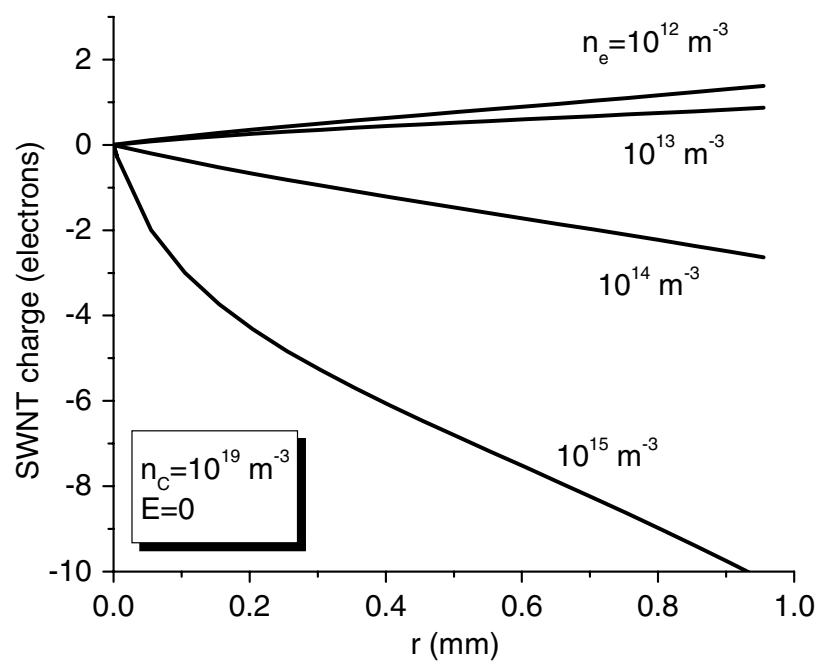

Figure 5. SWNT charge as a function of distance from the SWNT seed formation position with electron density as a parameter.

a parameter. We consider a case with low electron density (less than $10^{10} \mathrm{~m}^{-3}$ ). It can be seen (figure 3) that the SWNT charge is positive due to significant thermoionic emission. In addition the SWNT charge depends on the carbon density, which is a result of the SWNT growth rate dependence on the carbon species density. Higher carbon density leads to faster growth of the SWNT, as shown in figure 4, and thus causes larger total charge accumulated by SWNT. Recall that in the case of a large plasma density this model predicts the formation of SWNT with a very large aspect ratio.

SWNT charge dependence on the electron density is shown in figure 5 . One can see that a higher electron density leads to a negative SWNT charge due to strong electron flux from the plasma to the SWNT surface that exceeds thermoionic emission and ion flux. Thus, both negative and positive SWNT charge can be realized depending on the electron density in the SWNT formation region. Based on this prediction, different strategies of manipulation of SWNT growth by electric field can be implemented.

One possibility to affect SWNT growth in the SWNT formation region is to apply an electric field. Due to the fact that SWNT accumulates charge in the course of interaction with 


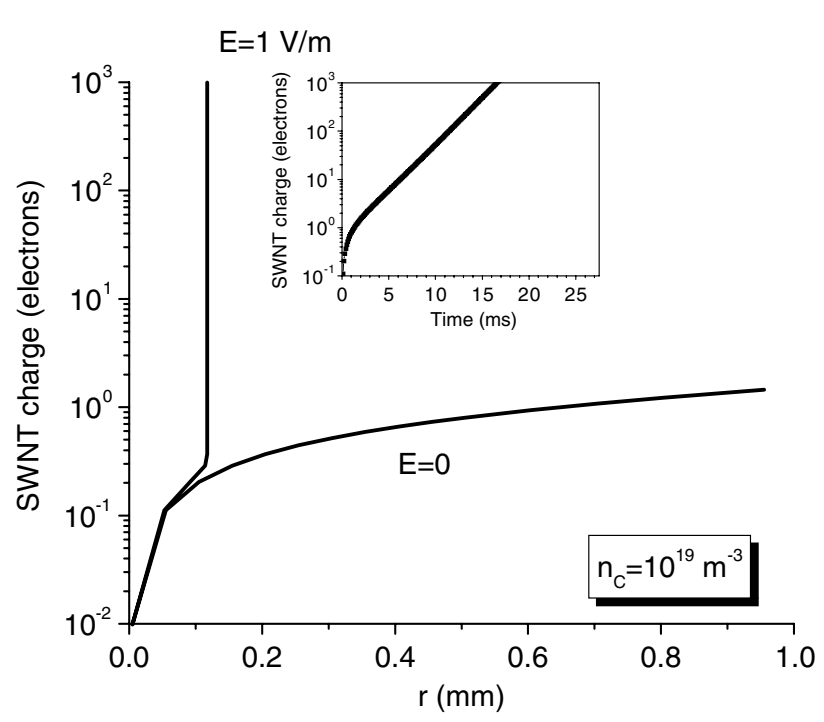

Figure 6. SWNT charge as a function of distance with electric field as a parameter.

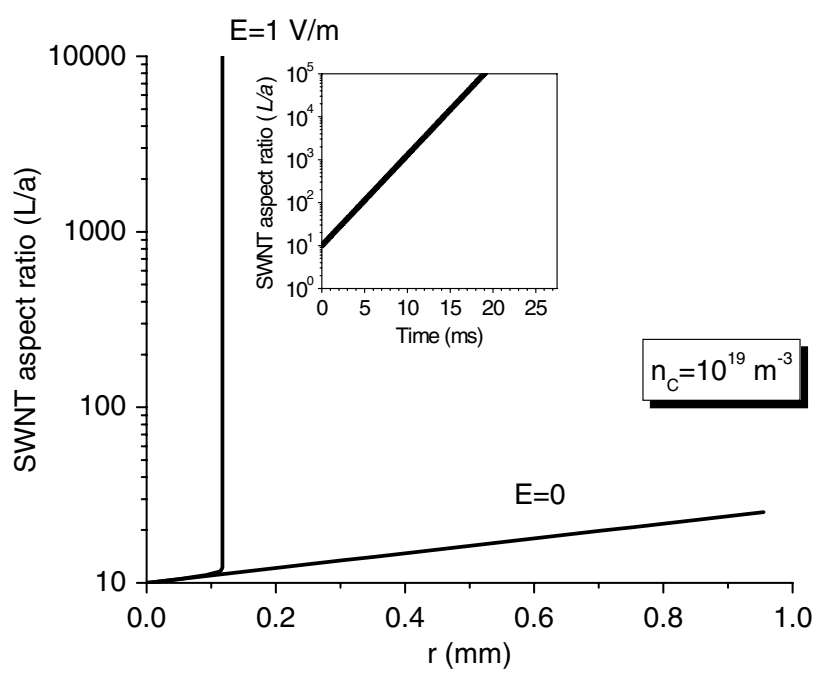

Figure 7. SWNT aspect ratio as a function of distance with electric field as a parameter.

plasma, it is expected that this electric field may affect SWNT motion. In fact our simulations show that the electric field in the SWNT formation region has a strong effect on SWNT motion. SWNT charge distribution is shown in figure 6 and SWNT relative length (aspect ratio) is shown in figure 7 with an electric field in the SWNT growth region as a parameter. It can be seen that a relatively small electric field significantly affects SWNT growth and leads to a large SWNT aspect ratio in comparison with the zero electric field case. This is due to trapping of SWNT in a region of the preferable growth. In the enlarged section of figures 6 and 7 one can see time evolution of the SWNT charge and the SWNT aspect ratio.

\section{Concluding remarks}

This model suggests several ways to improve the controllability of SWNT growth in the arc discharge. In particular, it is shown that the SWNT aspect ratio can be substantially altered during the arc discharge synthesis. Among the other possibilities increase in the plasma density and application of the electric field are the major factors. It should be pointed out that predicted parameters required for this could be implemented under typical arc conditions. Thus, it can be expected that some of these ideas can be translated into a practical application of the arc discharge for controlled SWNT synthesis.

Previous models suggested various scenarios for SWNT growth termination in arc discharge. These scenarios are tied to the mechanism of the carbon species precipitation and chemistry involved in the SWNT synthesis. For instance, in the framework of the growth model that explains the root-growth of nanotube bundles emerging from catalyst particles, catalyst phase diagram of carbon-metal ultimately affects the SWNT synthesis. However, in addition to that, in the dynamic system such as the arc discharge, the SWNT growth is a function of the SWNT seed motion along the discharge plasma. Due to momentum transfer from the flowing plasma, SWNT gains velocity and leaves the region of preferable formation. Thus, the time of residence in the area of preferable growth is limited. Therefore, increase in the SWNT residence time in that region may affect SWNT growth and may be considered as a way to control the SWNT parameters. In this paper we showed that application of an electric field has a profound effect on SWNT growth. In addition, it was predicted that the plasma density increase could also promote SWNT growth. As a possibility, application of an axial magnetic field can be used to enhance plasma density in the arc discharge. In fact, previous experimental and theoretical studies showed that significant increase in the plasma density could be achieved by applying a relatively moderate magnetic field [46].

Noteworthy, SWNT-plasma interactions lead to SWNT charging. This model predicts that both negative and positive charge can be accumulated by SWNT dependent on the plasma density. Thus, we want to emphasize that different polarities of the electric field should be implemented to affect the SWNT growth dependent on the plasma density.

\section{Acknowledgments}

The author thanks Liang Tao and Angela Knapp for helping with carbon nanotube preparation and University of Michigan Undergraduate Research Opportunity Program (UROP) for their support. He would also like to thank Anthony Waas and Yevgeny Raitses for very useful discussions.

\section{References}

[1] Lee S B, The A S, Teo K B K, Chhowalla M, Hasko D G, Milne W I, Amaratunga G A J and Ahmed H 2003 Nanotechnology 14192

[2] Buldum A and Lu J P 2003 Phys. Rev. Lett. 91 236801-1.

[3] Chhowalla M, Teo K B K, Ducati C, Rupesinghe N L, Amaratunga G A J, Ferrari A C, Roy D, Robertson J and Milne W I 2001 J. Appl. Phys. 905308

[4] Ostrikov K 2005 Rev. Mod. Phys. 77489

[5] Gao H, Wu X B, Li J T, Wu G T, Lin J Y, Wu K and Xu D S 2003 Appl. Phys. Lett, 833389.

[6] Kong J, Franklin N R, Zhou C, Chapline M G, Peng S, Cho K and Dai H 2000 Science 287622

[7] Levchenko I, Ostrikov K, Keidar M and Xu S 2005 J. Appl. Phys. 98064304 
[8] Levchenko I, Ostrikov K, Keidar M and Xu S 2006 Appl. Phys. Lett. 89033109

[9] Iijima S 1991 Nature, 35456

[10] Huang Z, Xu J, Ren Z, Wang J, Siegal M and Provencio P 1998 Appl Phys. Lett. 733845

[11] Wang Y H, Kim M J, Shan H W, Kittrell C, Fan H, Ericson L M, Hwang W F, Arepalli S, Hauge R H and Smalley R E 2005 Nano Lett. 5997

[12] Arepalli S 2004 J. Nanosci. Nanotech. 4317

[13] Okawa Y, Kitamura S and Iseki Y 2005 9th Spacecraft Charging Technology Conf. (Japan, April 2005)

[14] Moravsky A P, Waxler E M and Loutfy R O 2005 Growth of carbon nanotubes by arc discharge and laser ablation Carbon Nanotubes: Science and Applications ed M Meyyappan (Boca Raton, FL: CRC Press) pp 65-97

[15] Takikawa H, Yatsuki M, Sakakibara T and Itoh S 2000 J. Phys. D: Appl. Phys. 33826

[16] Waldorff E I, Waas A M, Friedmann P P and Keidar M 2004 J. Appl. Phys 952749

[17] Farhat S, de la Chapelle M L, Loiseau A, Scott C D, Lefrant S, Journet C and Bernier P 2001 J. Chem. Phys. 1156752

[18] Keidar M, Raitses Y, Knapp A and Waas A M 2006 Carbon 441022

[19] Journet C, Maser W K, Bernier P, Loiseau A, delaChapelle M L, Lefrant S, Deniard P, Lee R and Fischer J E 1997 Nature 388756

[20] Ando Y, Zhao X, Hirahara K, Suenaga K, Bandow S and Iijima S 2000 Chem Phys. Lett. 323580

[21] Zhao T and Liu Y 2004 Carbon 422735

[22] Lv X, Du F, Ma Y, Wu Q, Chen Y 2005 Carbon 432020

[23] Tang D, Sun L, Zhou J, Zhou W, Xie S 2005 Carbon 432812

[24] Yao M, Liu B, Zou Y, Wang L, Li D, Cui T, Zou G and Sundqvist B 2005 Carbon 432894

[25] Farhat S, de la Chapelle M L, Loiseau A, Scott C D, Lefrant S, Journet C and Bernier P 2001 J. Chem. Phys. 1156752
[26] Hinkov I, Farhat S and Scott C D 2005 Carbon 432453

[27] Bower C, Zhou O, Zhu W, Werder D J and Jin S 2000 Appl. Phys. Lett. 772767

[28] Wildoer J W G, Venema L C, Rinzler A G, Smalley R E and Dekker C 1998 Nature 39159

[29] McKelliget J W and Szekely J 1983 J. Phys. D: Appl. Phys. 161007

[30] Beilis I I 1999 IEEE Trans. Plasma Sci. 27821

[31] Lefort A, Parizet M J, El-Fassi S E and Abbaoui M 1993 J. Phys. D: Appl. Phys. 261239

[32] Bilodeau J F, Pousse J and Gleizes A 1998 Plasma Chem Plasma Proc. 18285

[33] Hinkov I, Grand J, de la Chapelle M L, Farhat S, Scott C D, Nikolaev P, Pichot V, Launois P, Mevellec J Y and Lefrant S 2004 J. Appl. Phys. 952029

[34] Keidar M, Waas A M, Raitses Y and Waldorff E 2006 J. Nanosci. Nanotechnol. 61309

[35] Farhat S and Scott C D 2006 J. Nanosci. Nanotechnol. 61189

[36] Keidar M and Waas A M, 2004 Nanotechnology 151571

[37] Zheng L X et al 2004 Nature Mater. 3673

[38] Gamaly E G and Ebbesen T W 1995 Phys. Rev. B 522083

[39] Iijima S, Ajayan P M and Ichihashi T 1992 Phys. Rev. Lett. 693100

[40] Saito Y, Yoshikawa T and Inagaki M 1993 Chem. Phys. Lett. 204277

[41] Colbert D T and Smalley R E 1995 Carbon 33921

[42] Zhou D and Chow L 2003 J. Appl. Phys. 939972

[43] Mieno T and Takeguchi M 2006 J. Appl. Phys. 99104301

[44] Winske D and Jones M E 1995 IEEE Trans. Plasma Sci. 23188

[45] Beilis I, Keidar M, Boxman R L, Goldsmith S, Heberlein J and Pfender E 1999 J. Appl. Phys. 86114

[46] Keidar M, Beilis I, Boxman R L and Goldsmith S 1996 J. Phys. D: Appl. Phys. 291973 